\title{
Oscillations in Pulse-Width Modulated System
}

\section{A.Kh. Gelig, N.V. Kuznetsov}

\author{
Draft $^{1}$
}

Abstract:- A system consisting of a continuous stable linear part and of a pulse element is considered, which realizes one of the following kinds of modulation: the integral pulse-width modulation, the linear integral pulse-width modulation with the modulation of the trailing edge of pulse, the linear integral pulse-width modulation with the modulation of the leading edge of pulse. Sufficient frequency conditions for existence of the forced periodic regime are obtained.

Keywords: Oscillations, Pulse-Width Modulated System

\section{Problem setting.}

Consider a system

$$
\dot{x}=A x+b f, \quad \sigma=c^{*} z+\psi(t),
$$

where $A$ is a constant Hurwitz's $k \times k$-matrix, $b$ and $c$ are constant $k$-dimensional columns, $\psi(t)$ is a given absolutely continuous $N T$ - periodic function ( $N$ is an integer number), $\sigma(t)$ is a signal on the input of the pulse element, $f(t)$ is a signal on its output. We shall study modulations of three kinds [1]: the integral pulsewidth modulation (IPWM), the linear integral pulse-width modulation with the modulation of the trailing edge pulse (LIPWM-1), and the linear pulse-width modulation with the modulation of the leading edge of pulse (LIPWM-2). In the case of IPWM and LIPWM-2 we have

$$
f(t)=\left\{\begin{array}{l}
0, \quad n T \leq t<n T+\nu_{n} \\
\lambda_{n}, \quad n T+\nu_{n} \leq t<(n+1) T
\end{array}\right.
$$

For LIPWM-1

$$
f(t)=\left\{\begin{array}{l}
\operatorname{sign} \sigma(n T), \quad n T<t \leq n T+\nu_{n} \\
0, \quad n T+\nu_{n} \leq t \leq(n+1) T
\end{array}\right.
$$

Here $T>0, \lambda_{n}=\operatorname{sign}\left(y_{n}\left(\nu_{n}\right)\right), y_{n}(\nu)=\int_{0}^{\nu} \sigma(n T+\tau) d \tau$. In the case IPWM $\left(\nu_{n}\right)$ is the first positive root of the following equation

$$
\left|y_{n}(\nu)\right|=\Delta
$$

if such a root exists on $(0, T]$ and $\nu_{n}=T$ in the opposite case. For LIPWM-1 and LIPWM-2 $\nu_{n}$ is a minimal root of the following equation

$$
\left|y_{n}(\nu)\right|=\Phi(\nu),
$$

where $\Phi(\nu)=\frac{\Delta \nu^{2}}{T^{2}}$ in the case LIPWM-1 and $\Phi(\nu)=\frac{\Delta \nu}{T}\left(1-\frac{\nu}{T}\right)$ in the case LIPWM-2.

Here we supposed that either

$$
|\sigma(n T)|>0
$$

\footnotetext{
${ }^{1}$ Nikolay V. Kuznetsov, nkuznetsov239 at gmail.com (correspondence author)
} 
for LIPWM-1 or

$$
|\sigma(n T)|<\Delta / T
$$

for LIPWM-2. If there is no such root, then $\nu_{n}=T$. When conditions (6),(7) are violated, we set $\nu_{n}=0$ and then for $n T \leq t<(n+1) T$ in place of $(2),(3) f(t)=0$ for LIPWM-1 and $f(t)=\operatorname{sign} \sigma(n \tau)$ for LIPWM-2.

A peculiarity of kinds of modulation considered is that the periodic regime in system (1) may be violated for certain values of parameters. The reason is that the continue dependence of the functional $\nu_{n}$ on the modulating signal $\sigma(t)$ is unavailable. Therefore the shift operator $S$ along trajectories is not continue in all space and the topological principle of fixed point for this operator is no applicable directly. To overcome this difficulty, in [1] an approach is suggested: to construct an invariant ellipsoid in the phase space such that the operator $S$ is continuous inside this ellipsoid. Developing this approach, we construct the invariant region of continuity for the operator $S$ bounded by a surface of the fourth order. As a result, new frequency conditions are obtained for existence of $N T$-periodic regime.

\subsection{Formulation of the result.}

Let us introduce the notation: $\Omega=N T, m=\min _{0 \leq t \leq \Omega} \psi, M=\max _{0 \leq t \leq \Omega} \psi, m_{0}=\inf _{0 \leq t \leq \Omega} \frac{d \psi}{d t}, M_{0}=\sup _{0 \leq t \leq \Omega} \frac{d \psi}{d t}, r=A^{*} c$, $\kappa=-c^{*} b, \beta_{0}=\min \left(\frac{\Delta}{T^{2}}-M_{0}, \frac{\Delta}{T^{2}}+m_{0}\right), \beta_{1}=\frac{\Delta}{T^{2}}+\kappa-\max \left(M_{0},-m_{0}\right), \beta_{2}=\beta_{1}-\kappa$.

Let

$$
\begin{gathered}
y=\operatorname{col}\left(x_{1}^{2}, x_{1} x_{2}, \ldots, x_{1} x_{k}, x_{2}^{2}, x_{2} x_{3}, \ldots,\right. \\
\left., x_{2} x_{k}, \ldots, x_{k-1}^{2}, x_{k-1} x_{k}, x_{k}^{2}\right) .
\end{gathered}
$$

Than it is obvious that $y \in \mathbf{R}^{l}, l=\frac{k(k+1)}{2}$. Differentiating the vector $y$ with respect to $t$, by virtue of system (1) and following [2], we obtain an equation

$$
\dot{y}=P y+\sum_{i=1}^{k} q_{i} \eta_{i},
$$

where $P, \eta_{i}=x_{i} f$ are a constant $l \times l$-matrix and constant $l$-dimensional columns. Define $l$-dimensional columns $s$ and $p$ from identities

$$
(r, s) \equiv(s, y),(x, x) \equiv(p, y),
$$

and consider functions $w_{j}=s^{*}(P-\lambda I)^{-1} q_{i}, g_{j}=p^{*}(P-\lambda I)^{-1} q_{i}$, where $I$ is a unit $l \times l$-dimensional matrix. Put $\beta=\beta_{0}$ in the case IPWM, $\beta_{1}$ in the case LIPWM-1, $\beta_{2}$ in the case LIPWM-2.

Theorem. Let a pair $\left(P, \sum_{i=1}^{k} q_{i} q_{i}{ }^{*}\right)$ be controllable and let there exists $\epsilon>0$ such that the matrix $P+\epsilon I$ is Hurwitz's one, $\beta>0$, and an inequality

$$
\begin{gathered}
{\left[\sum_{i=1}^{k} \int_{-\infty}^{+\infty}\left|w_{j}(i \omega)-\epsilon\right|^{2} d \omega\right]\left[\sum_{i=1}^{k} \int_{-\infty}^{+\infty}\left|g_{j}(i \omega)-\epsilon\right|^{2} d \omega\right]<} \\
<16 \varphi^{2} \epsilon^{2} \beta^{4}
\end{gathered}
$$

is satisfied. Then system (1) with any kind of modulations considered above (IPWM, LIPWM-1, LIPWM-2) has an NT-periodic solution.

The proof is based on constructing in the band of continuity of the operator $S$ an invariant domain $y * K^{-1} y<1$, where $K$ is a positive definite matrix defined by an expression

$$
K=\frac{\gamma^{2}}{2 r} \int_{0}^{\infty} e^{(P+\epsilon I) t} \sum_{i=1}^{k} e^{\left(P^{*}+\epsilon I\right) t} d t
$$


where $\gamma$ is a parameter satisfying the inequality

$$
\begin{gathered}
\frac{1}{4 \varphi \epsilon} \sum_{j=1} k \int_{-\infty}^{\infty}\left|g_{j}(i \omega-\epsilon)\right|^{2} d \omega< \\
<\gamma^{2}<4 \varphi \epsilon \beta^{4}\left[\sum_{j=1} k \int_{-\infty}^{\infty}\left|w_{j}(i \omega-\epsilon)\right|^{2} d \omega\right]^{-1}
\end{gathered}
$$

\subsection{Acknowledgements}

The work was supported in part by the programm "Russian Universities"

\section{References}

[1] A.Kh. Gelig, A.N. Churilov Stabylity and Oscillations of Nonlinear Pulse-Modulated System, Birkhauser, Boston-Basel-Berlin. 1998.

[2] A.I. Barkin, A.L. Zelentsovskii, and P.V. Pakshin, Absolute Stability of Determined and Stochastic Control System [in Russian], Moscow, 1992. 